\title{
Chronic Coronary Syndromes - the ESC Redefines the Concept of Stable Coronary Artery Disease
}

\author{
Diana Opincariu
}

"George Emil Palade" University of Medicine, Pharmacy, Science and Technology, Târgu Mureș, Romania

\section{CORRESPONDENCE}

Diana Opincariu Str. Gheorghe Marinescu nr. 50 540136 Târgu Mureș, Romania Tel: +40 372653100

E-mail: diana.opincariu@yahoo.ro

\section{CHANGING PERSPECTIVES: STABLE CORONARY ARTERY DISEASE MAY NOT BE VERY STABLE}

Coronary artery disease (CAD) is a chronic, progressive affliction that leads to atherosclerotic plaque development and progression within the epicardial coronary arteries. Being a dynamic process, CAD generally presents with a prolonged stable phase, which may suddenly become unstable and lead to an acute coronary event. Thus, the concept of stable CAD may be misleading, as the risk for secondary events continues to exist, despite the increasing use of optimal pharmacological therapies, lifestyle modifications, and myocardial revascularization procedures. ${ }^{1}$ The 2019 guidelines of the European Society of Cardiology (ESC) for CAD have introduced a concept that categorizes the disease according to its clinical presentation into acute or chronic coronary syndromes (ACS and CCS, respectively). Previously defined as stable angina pectoris, CCS includes nowadays a heterogeneous population with or without anginal symptoms, with or without prior acute coronary syndromes, and with or without previous coronary revascularization procedures. ${ }^{2}$

\section{LONG-TERM OUTCOMES IN CHRONIC CORONARY SYNDROMES}

Given the vast data available on the short- and long-term outcomes following ACS, there was a serious need for a large longitudinal study to provide presentday information on the characteristics, therapeutic management, and long-term evolution of the broad and heterogeneous spectrum of CCS. The CLARIFY registry (ProspeCtive observational LongitudinAl RegIstry oF patients with stable coronary arterY disease) is a multicentric study conducted between 2009 and 2010 in 45 countries, which included 32,703 patients with CCS, with a follow-up period of 5 years. The primary endpoint was the composite of cardiovascular death and non-fatal myocardial infarction (MI), and the secondary endpoints 
included, among other, all-cause mortality, stroke, hospitalization for heart failure, and coronary revascularization. ${ }^{3}$ The rate of the primary endpoint was $8 \%$ at 5 years. Furthermore, patients with prior MI and angina were more prone to developing the primary event (11.8\%) compared to those without angina. Another radical observation of the CLARIFY registry was that the CCS population has changed, in the sense that the prescription of secondary prevention measures are more frequently used regardless of the geographical area or the clinical profile, in comparison to preexistent surveys. ${ }^{3,4}$ Furthermore, the broad spectrum of CCS also includes patients without anginal symptoms, but with equivalent clinical signs for angina, and more important, patients with prior MI, with or without revascularization. CLARIFY has shown that angina was associated with poor outcomes at 5 years, but only in subjects with previous myocardial infarction, thus suggesting that this is a high-risk population, which requires more intense observation and follow-up. ${ }^{3}$

If previous registries on patients with stable angina did not take into account patients with a history of ACS or coronary revascularization, the current definition of CCS encompasses a more diverse clinical profile that takes into account the patient's medical history, as well as previously documented CAD. This is, therefore, in line with the secular trends of cardiovascular disease, in which the increasing number of percutaneous coronary revascularization procedures has led to a significant increase in the number of patients with coronary stents who are hospitalized due to heart failure or angina symptoms.

\section{DIAGNOSTIC APPROACH OF CCS - THE CONCEPT OF CLINICAL LIKELIHOOD FOR CAD}

The first diagnostic step in CCS is to identify patients with unstable coronary events, based on the clinical presentation. The 2019 ESC guidelines have changed the pretest probability for CAD and have introduced the concept of "clinical likelihood of CAD" based on gender, age, clinical presentation, as well as medical history and prior investigations. ${ }^{2}$ The clinical likelihood of CAD will dictate further noninvasive testing and thus, will allow a more cost-effective diagnostic and therapeutic approach. ${ }^{5}$ The evaluation of left ventricular function is of essence in all patients with suspicion of CAD and should be achieved with the use of transthoracic echocardiography, as a class I indication, but cardiac magnetic resonance imaging may also be used in patients with an inconclusive echo study (class IIB indication, level of evidence C). ${ }^{2}$ Subjects with a high clinical likelihood of CAD should be referred to invasive coronary angiogram, but in patients with a low to intermediate pretest probability, noninvasive imaging is the diagnostic method of choice. In the previous guidelines (2013), the ECG stress test was used as an initial diagnostic test for stable CAD in patients with angina and an intermediate pre-test probability. ${ }^{6}$ The current CCS guidelines recommend, as a class I indication (level of evidence B), the use of coronary CT angiography (CCTA) as initial diagnostic test in patients where clinical assessment alone cannot exclude the presence of significant coronary atherosclerotic involvement. $2,7,8$

\section{NON-INVASIVE THERAPEUTIC MANAGEMENT}

The 2019 ESC guidelines focus, as the previous ones, on reducing symptoms and improving long-term prognosis by lifestyle modification and control of risk factors. ${ }^{2,6}$ By employing healthy lifestyle changes, the risk of adverse cardiovascular events will be significantly reduced. Such behavioral modifications should be used in addition to appropriate pharmacological and/or interventional therapies in patients with CCS. In such extent, there is a need for a multidisciplinary approach in patients with CAD, that could provide support in dietary advice for weight management, cognitive and behavioral therapies, advice and nicotine replacement therapies for smoking cessation, physical and exercise education, as well as cardiac rehabilitation programs for secondary prevention. ${ }^{9,10}$

The pharmacological treatment in CCS aims to prevent and reduce the frequency of anginal symptoms, as well as to decrease the rate of major adverse events. Anti-ischemic therapies should be individualized according to patient comorbidities, associated treatments, tolerance, and adherence. The lack of compliance to non-pharmacological and pharmacological cardiological therapies has been associated with $9 \%$ of cardiovascular events in Europe. ${ }^{11}$ Endorsing lifestyle modifications and adherence to medication as a part of each clinical visit is an important step in the management of patients with CCS.

Similarly to the previous guidelines, ${ }^{6}$ beta blockers and calcium channel antagonists remain the first line of treatment in stable CAD, despite the lack of any randomized clinical trial that compares this pharmacological approach with an alternative one. Associated use of short-active nitrates in acute effort angina or the use of long-acting nitrates for the prevention of anginal episodes with a 10-14h nitrate-free interval is recommended as a second line of treatment. ${ }^{12}$ Antithrombotics play an essential role in the secondary preventive strategies, as stable CAD often presents with unstable episodes caused by plaque erosion and 
thrombosis. Antiplatelets should be prescribed in view of the ischemic and hemorrhagic risk. Aspirin 75-100 mg daily is recommended in patients with a history of MI or revascularization, and it may be used in selected cases without any prior MI or PCI, but with definitive evidence of coronary lesions on imaging. As an alternative to aspirin, in patients with intolerance or with a higher bleeding risk, clopidogrel $75 \mathrm{mg}$ daily may be considered. Ticagrelor and prasugrel as alternative antiplatelet therapies have yet to have proven their safety and efficacy in patients with CCS. Low-dose rivaroxaban in association with aspirin for patients in sinus rhythm has also been proven effective in reducing the rate of ischemic events, at the expense of increased rates of non-fatal bleeding, as shown by the COMPASS trial, but the risk reduction was observed in a subgroup of patients with increased ischemic burden such as diabetes, peripheral artery disease, or chronic kidney disease. In patients that require oral anticoagulation, it is advised to use NOACs in preference to vitamin $\mathrm{K}$ antagonists. ${ }^{2,13-15}$ Other class I recommendations for pharmacological treatment include statins for all CCS subjects, and angiotensin converting enzyme inhibitors in case of heart failure, high blood pressure, or diabetes. ${ }^{2}$

Irrespective of the initial pharmacological strategy, it is of great importance to evaluate the response to therapy after 2-4 weeks from treatment initiation.

\section{CORONARY REVASCULARIZATION IN CCS - TO REVASCULARIZE OR NOT?}

Myocardial revascularization was recommended by the previous guidelines if optimal medical treatment did not promote relief of symptoms and if it may lead to improved prognosis. ${ }^{6,16}$

Currently, there is conflicting evidence on myocardial revascularization in CCS, derived from previous clinical trials and meta-analyses. Some state that revascularization (percutaneous or via coronary artery by-pass grafting) can lead to relief of symptoms, decreased use of anti-ischemic medication, and increased exercise capacity and quality of life. Moreover, it has also been shown that by revascularizing the coronary lesions that produce myocardial ischemia (as assessed via noninvasive imaging or invasive FFR $<0.8$ ), there is a significant long-term decrease of urgent revascularization or myocardial infarction compared to pharmacological treatment alone. ${ }^{17-19}$ Other studies, such as ORBITA (Objective Randomised Blinded Investigation with optimal medical Therapy or Angioplasty in stable angina), have shown no or modest additional benefits of $\mathrm{PCI}$ associated with optimal medical therapies in CCS. ${ }^{20-22}$
The results of the ISCHEMIA trial (International Study of Comparative Health Effectiveness With Medical and Invasive Approaches) have not been published before the release of the new CCS guidelines in September 2019. The study included 5,179 patients with CCS, with moderate-tosevere ischemia as proved by stress imaging, without left main stenosis or heart failure, with controlled symptoms by use of optimal medical treatment. The enrolled subjects were randomized to invasive or conservative treatment. The results revealed that at a median 3.3 years of follow-up there were no significant differences between the invasive and optimal pharmacological treatment alone for the composite of cardiovascular death, MI, hospitalization for unstable angina, heart failure, or cardiac arrest (13.3\% versus $15.5 \%$, HR $0.93, \mathrm{p}=0.34$ ). Quality of life was, however, significantly improved in the invasive group, with lowered frequency of angina and increased effort capacity. ${ }^{23}$

Nevertheless, the current class I indication (level of evidence A) is to perform myocardial revascularization in case of refractory angina after optimization of medical treatment and if there is noninvasive evidence of ischemia ( $>10 \%$ of the left ventricle), or abnormal invasive fractional flow reserve, or decreased left ventricular ejection fraction caused by ischemia. ${ }^{2,16}$ In all cases, in light of the current supporting evidence, different modalities of myocardial revascularization should be applied in addition to optimal medical therapies, tailored according to related procedural risk and its aftermath (dual antiplatelet treatment and subsequent bleeding risk), as well as taking into account the patients' preference.

In conclusion, the current ESC guidelines for stable coronary artery disease have radically changed the way we look at this disorder, which is actually not stable, but is characterized by a continuous evolution, with stable and unstable periods. While the pharmacological management has not been substantially modified, the introduction of the clinical likelihood approach had led to an increase in the number of patients that are less likely to have obstructive coronary atherosclerosis. For those patients, the use of coronary CTA is becoming more and more frequent. The current approach is to provide a more individualized therapeutic management in order to ensure an increased adherence to not only medication, but also to lifestyle changes, because, in the end, this is a chronic condition, with an increased related risk for adverse events and decreased quality of life.

\section{CONFLICT OF INTEREST}

Nothing to declare. 


\section{REFERENCES}

1. Fox KAA, Metra M, Morais J, Atar D. The myth of 'stable' coronary artery disease. Nat Rev Cardiol. 2020;17:9-21. Epub 2019 Jul 29.

2. Knuuti J, Wijns W, Saraste A, et al. 2019 ESC Guidelines for the diagnosis and management of chronic coronary syndromes. Eur Heart J. 2020;41:407-477.

3. Sorbets E, Fox K, Elbez Y, et al. Long-term outcomes of chronic coronary syndrome worldwide: insights from the international CLARIFY registry. Eur Heart J. 2019;00:1-10.

4. Daly CA, De Stavola B, Sendon JL, et al. Predicting prognosis in stable angina-results from the Euro heart survey of stable angina: prospective observational study. BMJ. 2006:332:262-267.

5. Juarez-Orozco LE, Saraste A, Capodanno D, et al. Impact of a decreasing pre-test probability on the performance of diagnostic tests for coronary artery disease. Eur Heart J Cardiovasc Imaging. 2019; 20:1198-1207.

6. Montalescot G, Sechtem U, Achenbach S, et al. 2013 ESC guidelines on the management of stable coronary artery disease: the Task Force on the management of stable coronary artery disease of the European Society of Cardiology. Eur Heart J. 2013;34:2949-3003.

7. SCOT-HEART investigators. CT coronary angiography in patients with suspected angina due to coronary heart disease (SCOT-HEART): an openlabel, parallel group, multicentre trial. Lancet. 2015;385:2383-2391.

8. Knuuti J, Ballo H, Juarez-Orozco LE, et al. The performance of noninvasive tests to rule-in and rule-out significant coronary artery stenosis in patients with stable angina: a meta-analysis focused on post-test disease probability. Eur Heart J. 2018:39:3322-3330.

9. Piepoli MF, Hoes AW, Agewall S, et al. The Sixth Joint Task Force of the European Society of Cardiology and Other Societies on Cardiovascular Disease Prevention in Clinical Practice (constituted by representatives of 10 societies and by invited experts) Developed with the special contribution of the European Association for Cardiovascular Prevention \& Rehabilitation (EACPR). Eur Heart J. 2016:37:2315-2381.

10. Booth JN III, Levitan EB, Brown TM, Farkouh ME, Safford MM, Muntner P. Effect of sustaining lifestyle modifications (nonsmoking, weight reduction, physical activity, and mediterranean diet) after healing of myocardial infarction, percutaneous intervention, or coronary bypass (from the REasons for Geographic and Racial Differences in Stroke Study). Am J Cardiol. 2014;113:1933-1940.
11. Chowdhury R, Khan $\mathrm{H}$, Heydon E, et al. Adherence to cardiovascular therapy: a meta-analysis of prevalence and clinical consequences. Eur Heart J. 2013;34:2940-2948.

12. Ferrari R, Pavasini R, Camici PG, et al. Anti-anginal drugs-beliefs and evidence: systematic review covering 50 years of medical treatment. Eur Heart J. 2019;40:190-194

13. Valgimigli M, Bueno H, Byrne RA, et al. 2017 ESC focused update on dual antiplatelet therapy in coronary artery disease developed in collaboration with EACTS: The Task Force for dual antiplatelet therapy in coronary artery disease of the European Society of Cardiology (ESC) and of the European Association for Cardio-Thoracic Surgery (EACTS). Eur Heart J. 2018;39:213-260

14. Orme RC, Parker WAE, Thomas MR, et al. Study of two dose regimens of ticagrelor compared with clopidogrel in patients undergoing percutaneous coronary intervention for stable coronary artery disease (STEEL-PCI). Circulation. 2018:138:1290-1300.

15. Eikelboom JW, Connolly SJ, Bosch J, et al. Rivaroxaban with or without aspirin in stable cardiovascular disease. N Engl J Med. 2017:377:1319-1330.

16. Neumann FJ, Sousa-Uva M, Ahlsson A, et al. 2018 ESC/EACTS Guidelines on myocardial revascularization. Eur Heart J. 2019;40:87-165.

17. Xaplanteris P, Fournier S, Pijls NHJ, et al. Five-year outcomes with PCl guided by fractional flow reserve. N Engl J Med. 2018;379:250-259.

18. Velazquez $\mathrm{EJ}$, Lee $\mathrm{KL}$, Jones $\mathrm{RH}$, et al. Coronary-artery bypass surgery in patients with ischemic cardiomyopathy. N Engl J Med. 2016;374:1511-1520.

19. Windecker S, Stortecky S, Stefanini GG, et al. Revascularisation versus medical treatment in patients with stable coronary artery disease: Network meta-analysis. BMJ. 2014;348:g3859

20. Al-Lamee R, Thompson D, Dehbi HM, et al. Percutaneous coronary intervention in stable angina (ORBITA): a doubleblind, randomised controlled trial. Lancet. 2018;391:31-40.

21. Stergiopoulos K, Brown DL. Initial coronary stent implantation with medical therapy vs medical therapy alone for stable coronary artery disease: metaanalysis of randomized controlled trials. Arch Intern Med. 2012:172:312-319.

22. Bangalore S, Pursnani S, Kumar S, Bagos PG. Percutaneous coronary intervention versus optimal medical therapy for prevention of spontaneous myocardial infarction in subjects with stable ischemic heart disease. Circulation. 2013;127:769-781.

23. International Study of Comparative Health Effectiveness With Medical and Invasive Approaches (ISCHEMIA). https://professional.heart.org/ professional/ScienceNews/UCM_505226_ISCHEMIA-Clinical-TrialDetails.jsp (06 December 2019) 